Conclusion Adherence clubs, family support and low stigma and discrimination are key factors to improve treatment adherence for people living with HIV and other non-communicable diseases. Interventions to increase family support and reduction in stigma and discrimination are encouraged.

\section{P4.76 HOW DO I NOTIFY PARTNERS? CHALLENGES FACED BY HEALTHCARE PROVIDERS MANAGING SYPHILIS IN CHILE}

${ }^{1}$ Nicole Iturrieta, ${ }^{1}$ Meredith Temple-Smith, ${ }^{2}$ Jane Tomnay. 'Department of General Practice University of Melbourne, Melbourne, Australia; ${ }^{2}$ Department of Rural Health - Centre for Excellence in Rural Sexual Health - University of Melbourne, Melbourne, Australia

\subsection{6/sextrans-2017-053264.571}

Introduction Partner Notification (PN) has long been considered an essential strategy for sexually transmitted infection (STI) control, but both policies and methods of implementation vary between countries. In Chile, syphilis is the most commonly reported STI in the general population; however, congenital syphilis is close to being eliminated. In this study, we explore the challenges faced by healthcare providers (HCP) when they perform PN for syphilis in public health services in Chile.

Methods To identify the nuances of delivering PN, semi-structured face-to-face interviews were conducted with HCP. A third of the interviews were transcribed verbatim and translated from Spanish to English for thematic analysis, which followed an inductive approach based on grounded theory. Following the identification of themes, remaining interviews were coded utilising constant comparison.

Results 48 HCP were interviewed in PHC centres and sexual health units. The primary challenge revealed by HCP in this study was the low level of awareness about STI risk and potential long-term implications for patients. HCP recognised that a significant lack of recognition of $\mathrm{PN}$ for the value of STI control, and consequently it is not undertaken routinely. Index patients are reluctant to reveal partner information, likely to avoid perceived stigma. Furthermore, HCP identified several limitations within the Chilean system, such as lack of available counselling for patients, absence of resources for provider referral, and insufficient training and specialist support for effective PN.

Conclusion Improvements in syphilis management have achieved a significant reduction in congenital syphilis. However, our findings suggest syphilis elimination could be assisted by improved PN. Hence, it is vital to develop clear referral pathways for HCP to perform PN at the grassroots level.

\section{P4.77 SOCIAL-MEDIA USE AND SEXUAL BEHAVIOUR AMONG IN-SCHOOL ADOLESCENTS IN IBADAN, NIGERIA}

Oladipupo Samuel Olaleye. Department of Health Promotion and Education, College of Medicine, University of Ibadan, Nigeria, Ibadan, Nigeria

\subsection{6/sextrans-2017-053264.572}

Introduction Adolescence is a developmental period characterised by various opportunities including the social media. Adolescence is also embedded with several challenges that are dangerous to sexual health. In Nigeria, research focusing on the effects of social media on adolescents' sexual behaviour (SB) is very rare. This study was therefore designed to determine the use social media (SM) and its effects on SB of in-school adolescents in Ibadan, Nigeria.

Methods A cross-sectional study was carried out among randomly selected 194 senior secondary students in four secondary schools in Ibadan. The questionnaire explored the sociodemographic data, SB, frequency of use of SM, and activities on SM. Data was analysed using descriptive statistics and Chisquare at 0.05 level of significance.

Results The mean age of the respondents was $14.7 \pm 1.2$ and $50.3 \%$ were females. Some $(15.2 \%)$ reported ever experienced sexual intercourse. The mean age of sexual debut was 12.2 \pm 2.8 . Most (88.0\%) reported the use SM, the highest used social medium was Facebook (71.2\%) followed by Whatsapp $(55.0 \%)$ while the most frequently used was Whatsapp followed by facebook; $44.7 \%$ and $25.4 \%$ reported everyday use respectively. Activities reported on SM included reading of news $(60.7 \%)$, download/listen to music $(62.8 \%)$, watch/download pornographic pictures $(5.2 \%)$, watch/download pornographic videos $(8.4 \%)$, search for sexual partners $(16.8 \%)$, share pornographic pictures (4.2\%) and videos (4.7\%). Significantly, more of those who had ever experienced sexual intercourse watched/downloaded pornographic pictures $(24.1 \%$ vs $1.9 \%)$ and videos $(37.9 \%$ vs $3.1 \%)$, and search for sexual partners $(58.6 \%$ vs $9.3 \%)$ using the SM.

Conclusion A number of the adolescents use social-media and many engage in activities that could be risky to their sexual life on these media especially those who have ever experienced sexual intercourse. Intervention to ensure safe use of the social-media should be carried out among these young persons.

\section{P4.78 USING A SYSTEMS APPROACH TO DESIGN HIGHER- IMPACT INTERVENTIONS: ILLUSTRATING LEVELS OF LEVERAGE FOR REDUCING CONCURRENCY-LINKED SEXUALLY TRANSMITTED INFECTIONS IN AFRICAN AMERCIAN ADOLESCENTS FROM DISADVANTAGED COMMUNITIES}

${ }^{1}$ Pamela A Matson, ${ }^{2}$ Andra Wilkinson, ${ }^{3}$ Kristen Hassmiller Lich, ${ }^{4}$ Jonathan Mark Ellen, ${ }^{3}$ Seri Link Anderson. 'Johns Hopkins University School of Medicine, Baltimore, USA; ${ }^{2}$ Child Trends, Chapel Hill, USA; ${ }^{3}$ University of North Carolina Gillings School of Public Health, Chapel Hill, USA; ${ }^{4}$ Johns Hopkins All Children Hospital, St. Petersburg, USA

\subsection{6/sextrans-2017-053264.573}

Introduction High sexually transmitted infection (STI) rates among African American adolescents living in disadvantaged communities represent a complex public health problem that has remained unsolved despite the efforts of governments (both state and federal) and communities. Systems thinking is an approach that can give new insight into why previous attempts to tackle this problem have failed and how to design more impactful and sustainable interventions in the future. In this paper, we introduce a system dynamics framework that describes increasingly impactful levels of leverage that public health actions might target to reduce incidence of sexually transmitted infections among adolescents in disadvantaged communities. Then, we use the levels of leverage framework to describe why previous interventions to reduce high STI rates among African American adolescents from disadvantaged communities have had minimal success as well as to suggest some interventions that are likely to have a higher impact. We propose several previously overlooked targets for interventions that are aligned with existing feedback loops affecting STI 
outcomes, and provide substantial opportunities for creating long-lasting reductions in STI rates.

\section{P4.79 THE DISTRIBUTION OF SEX PARTNERS IN THE UNITED STATES BY SEXUAL IDENTITY: DATA FROM THE NATIONAL SURVEY OF FAMILY GROWTH}

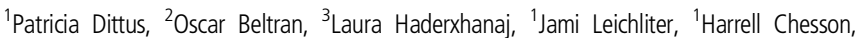
${ }^{1}$ Sevgi Aral. ${ }^{1}$ Centres for Disease Control and Prevention, Atlanta, USA; ${ }^{2}$ OAK Ridge Institute for Science and Education, Atlanta, USA; Indiana University Bloomington, Bloomington, USA

\subsection{6/sextrans-2017-053264.574}

Introduction Risky sexual behaviour and STIs cluster within subpopulations. Examining the concentration of behaviours can help to target interventions to those at highest risk. Given STI disparities by sexual identity, we examined the distribution of sexual partners among heterosexual, homosexual, and bisexual males and females.

Methods Data from the 2006-15 U.S. National Survey of Family Growth were used to analyse the number of oppositesex and same-sex partners in the past 12 months, focusing on means and the percentage of sex partners accounted for by the top $20 \%$ of each subpopulation; $95 \%$ confidence intervals were used to examine significant differences. Demographics will also be reported.

Results Bisexual women reported a higher mean number of recent opposite-sex partners $(1.81 ; 95 \% \mathrm{CI}: 1.66,1.96)$ than heterosexual $(1.21 ; 95 \% \mathrm{CI}: 1.19,1.24)$ and homosexual $(0.79$; 95\% CI: $0.26,1.32)$ women, whereas homosexual women reported a higher number of same-sex partners (1.55; 95\% CI: $1.39,1.72)$ than bisexual $(0.74 ; 95 \% \mathrm{CI}: 0.64,0.84)$ and heterosexual women $(0.21 ; 95 \% \mathrm{CI}: 0.17,0.25)$. Within each subgroup, the top $20 \%$ of bisexual and heterosexual women accounted for $45 \%$ and $37.6 \%$ of opposite-sex partners, respectively. Among males, there was no difference in the mean number of recent opposite-sex partners between heterosexual and bisexual men (1.5 partners), whereas homosexual men reported a significantly higher mean number of recent same-sex partners $(2.39 ; 95 \% \mathrm{CI}: 2.11,2.67)$ than bisexual (1.16; 95\% CI: $0.87,1.45)$ and heterosexual men $(0.18 ; 95 \% \mathrm{CI}: 0.12,0.24)$. Within each subgroup, the top $20 \%$ of heterosexual men accounted for $47.1 \%$ of oppositesex partners compared to the top $20 \%$ of gay and bisexual men accounting for $48.1 \%$ and $59.8 \%$ of same-sex partners, respectively.

Conclusions In general, sexual minorities reported higher numbers of recent sex partners, however, interesting gender differences emerged, particularly with regard to the top $20 \%$ of subpopulations. Interventions targeting high-risk persons within sub-populations may be more impactful.

\section{P4.80 EMPOWERING ADOLESCENTS THROUGH ART EDUCATION FOR SEXUAL HEALTH PROMOTION}

${ }^{1}$ Patricia Neyva da Costa Pinheiro, ${ }^{2}$ Anny Gisely Milhome da Costa Farre, ${ }^{1}$ Fabiane do Amaral Gubert, ${ }^{1}$ Neiva Francenely Cunha Vieira. ${ }^{1}$ Universidade Federal do Ceará, Fortaleza - CE, Brazil; ${ }^{2}$ Universidade Federal de Sergipe, Lagartos - SE, Brazil

10.1136/sextrans-2017-053264.575
Introduction The objective of this study was to evaluate the contributions of Art Education for sexual health promotion of adolescents in the context of urban social vulnerability, through the process of consciousness and empowerment.

Methods Community-based participatory research developed with 21 adolescents who were participating in a governmental program of Art Education and resocialization located in a Brazilian poor community. Data were collected in five months by Fetterman's method of Empowerment Evaluation through weekly group meetings and participant observation by critical friends. Data analysis was supported by Freire's concepts of Critical Consciousness with a qualitative approach to data categorization.

Results Adolescents oriented their needs and priorities of health into two main themes and their categories: 1) Community: health care, diseases, trafficking, violence, drugs and leisure; 2) Adolescence: body, mind, friendships, dating, sex and fun. The Art Education activities - visual, audiovisual and dance - were articulated with reflections about these subjects and showed the power of community relationships in sexual health promotion of adolescents. Some changes were observed after reevaluation of the process and involved the triad: individual, community and program. A predominantly negative view of the neighbourhood was becoming a vision full of possibilities, examples and personal empowerment for health and wisely choices.

Conclusion This research proposed a model of Adolescent Health Promotion Based on Art Education and CommunityCentred. The group walked towards intransitive consciousness, for the transitive-naive and critical, featuring an awareness process that began with the seizure of a health reality initially considered fateful and immutable, past the living situations that favoured the questioning of this reality and culminated with the perception of the possibilities of change and selfdetermination for change.

\section{P4.81 ADOLESCENT CHAT: MOBILE APPLICATION ON SEXUALITY, STD/HIV/AIDS PREVENTION}

${ }^{1}$ Patricia Neyva da Costa Pinheiro, ${ }^{2}$ Mariana Borges Sodré, ${ }^{3}$ Thailane Maria Silva Santana, ${ }^{2}$ Adrian Gomes Nogueira Ferreira, ${ }^{4}$ Geraldo Braz Junior. 'Universidade Federal do Ceará, Fortaleza - CE, Brazil; ${ }^{2}$ Universidade Federal do Maranhão, Imperatriz - MA, Brazil; ${ }^{3}$ Universidade Federal do Maranhão, Imperatiz - Ma, Brazil; ${ }^{4}$ Universidade Federal do Maranhão, São Luis - MT, Brazil

\subsection{6/sextrans-2017-053264.576}

Introduction The popularisation of mobile devices has been considered by many to be the technological revolution of greatest impact in recent years. Thus, the development of computational solutions in the form of applications for mobile devices represents an effective means of making available health education contents for adolescents. The objective of the study was to describe the development of the application Adolescent Chat with information on sexuality and STD/HIV/ AIDS prevention.

Methods This is a research and methodological development, carried out in 2016. In this study, an application was developed based on the website already developed, Adolescent Chat, containing subjects on sexuality and STD/HIV/AIDS prevention as a tool to promote Adolescents that attend the Catholic Church. Ethical aspects were respected in accordance with protocol No. 1.165.118. 